\title{
Niveles de calcio para codornices en postura
}

\author{
Calcium levels in layers quail
}

Níveis de cálcio para codornas em postura

Víctor L. Hurtado- Nery ${ }^{1 *}$, Jonny Fabiola Guevara-Paez ${ }^{2 *}$, Dumar J. Forero-Osuna ${ }^{2 *}$

1 MVZ, MSc, PhD, Facultad de Ciencias Agropecuarias y Recursos Naturales, Universidad de los Llanos;

2 MVZ, Universidad de los Llanos

* Grupo de Estudios en Nutrición Animal Universidad de los Llanos, km 12 Vía Apiay, Villavicencio, Colombia.

Email:vhurtado@unillanos.edu.co

Recibido: junio 11 de 2015

Aceptado: septiembre 20 de 2017

\begin{abstract}
Resumen
Con el objetivo de evaluar el efecto de los niveles de calcio en la dieta sobre los parámetros productivos de codornices, fue realizado este trabajo en la Unidad de codornices de la Universidad de los Llanos, con 240 codornices de 75 días de edad y 151,9 $\pm 8,5 \mathrm{~g}$ de peso, durante 26 semanas, distribuidas en un diseño experimental completamente al azar, con cuatro tratamientos, seis repeticiones y 10 aves por repetición, para un total de 24 unidades experimentales. Las aves fueron alojadas en jaulas de alambre, en módulos de cinco pisos con tres divisiones por piso, dotadas de comederos lineales y bebederos automáticos. Los tratamientos fueron elaborados con inclusiones de carbonato de calcio $(4,9 ; 5,28 ; 5,67$ y $6,06)$ para constituir dietas con niveles de 2,$30 ; 2,45 ; 2,60$ y 2,75\% de calcio. Los niveles de calcio influyeron $(p<0,05)$ la producción de huevos (80,19; 81,76; 71,67 y 79,15\%), el consumo diario de ración (24,26; 24,76; 24,50 y 24,53 g/ave/ día), la conversión alimenticia por docenas de huevo $(0,333 ; 0,340 ; 0,389$ y 0,347) y la conversión por masa de huevo $(2,77,2,84,3,24$ y 2,89). Sobre el peso del huevo $(10,91 ; 10,69 ; 10,55$ y 10,74 g) no hubo efectos (P>0,05). En conclusión, el nivel de calcio que permite obtener mejores parámetros productivos se estima en 2,48\%.
\end{abstract}

Palabra clave: Coturnix coturnix, minerales, postura, requerimientos nutricionales

\begin{abstract}
In order to evaluate the effect of calcium levels in the diet on performance of quails it was performed this work in quail Unit of the University of the Llanos, with 240 quails 75 days old and $151.9 \pm 8.5$ g weight for 26 weeks, distributed in a completely randomized experimental design, with four treatments, six replications and 10 birds per repetition, for a total of 24 experimental units. The birds were housed in wire cages, five-storey modules with three divisions per floor, equipped with linear feeders and automatic drinkers. Treatments were made by inclusions of limestone (4.9; 5.28; 5.67 and 6.06 ) for diets $2.30 ; 2.45 ; 2.60$ and $2.75 \%$ calcium. Calcium levels influenced egg production (80.19, 81.76, 71.67 and $79.15 \%)$, daily feed intake $(24.26 ; 24.76 ; 24.50$ and $24.53 \mathrm{~g} /$ bird / day), feed conversion per dozen eggs $(0.333,0.340,0.389$ and 0.347$)$ and mass conversion Egg $(2.77,2.84,3.24$ and 2.89) on egg weight (10.91; 10.69; 10.55 and $10.74 \mathrm{~g}$ ) there was no effect ( $\mathrm{P}>0 \mathrm{05}$ ). In conclusion, the level of calcium that leads to better production parameters is $2.48 \%$.
\end{abstract}

Keywords: Coturnix coturnix, minerals, egg production, nutrients requirements. 


\begin{abstract}
Resumo
Para avaliar o efeito dos níveis de cálcio na dieta sobre os parâmetros produtivos de codornas, este trabalho foi realizado na Unidade de Codornas da Universidade dos Llanos, com 240 codornas de 75 dias de idade e 151,9 \pm 8,5 g de peso, durante 26 semanas, distribuídos em delineamento experimental inteiramente casualizado, com quatro tratamentos, seis repetições e 10 aves por repetição, para um total de 24 unidades experimentais. As aves foram alojadas em gaiolas de arame, em módulos de cinco andares com três divisões por andar, equipadas com comedouros lineares e bebedouros automáticos. Os tratamentos foram elaborados com inclusões de carbonato de cálcio (4.9, 5.28, 5.67 e 6.06) para formar dietas com níveis de 2,30; 2,45; 2,60 e 2,75\% de cálcio. Os níveis de cálcio influenciaram ( $\mathrm{p}<0,05)$ a produção de ovos $(80,19,81,76,71,67$ e 79,15\%), consumo diário de ração $(24,26,24,76,24,50$ e 24,53 g / ave / dia), a conversão alimentar por dúzia de ovos $(0,333,0,340,0,389$ e 0,347$)$ e a conversão por massa de ovo $(2,77,2,84,3,24$ e 2,89$)$. No peso do ovo $(10,91,10,69,10,55$ e 10,74 g), não houve efeitos (P> 0,05). Em conclusão, o nível de cálcio que permite obter melhores parâmetros produtivos é estimado em 2,48\%.
\end{abstract}

Palavra chave: Coturnix coturnix, minerais, postura, requisitos nutricionais

\section{Introducción}

Las codornices representan una alternativa para la alimentación humana, estas aves se utilizan para la producción de huevos y carne, con aceptación universal por ser un producto de alta calidad (Fugikura, 2002). El suministro de los requerimientos nutricionales en las dietas de codornices, busca que las aves expresen su máximo potencial genético para la producción de huevos, se considera la exigencia de un nutriente la cantidad precisa a ser ofrecida en la dieta para atender las necesidades del animal en condiciones de un ambiente compatible con la buena salud del animal (Sakomoura y Rostagno, 2007).

Los requerimientos nutricionales varían según la edad, sexo, temperatura ambiente, consumo de ración, línea genética, etc., para controlar estas fuentes de variación, se debe definir los niveles a ser evaluados para obtener la respuesta en el rendimiento animal. En este sentido, el calcio y el fósforo son los minerales más importantes en la nutrición animal, porque influyen no solo sobre el crecimiento, sino también por ser los principales formadores de la matriz mineral, los cuales deben estar en la dieta en cantidades y proporciones adecuadas para atender las exigencias de fósforo y calcio de los animales en función de la edad, la raza, la condición fisiológica y el sistema de producción adoptado (Silva et al., 2009).

Durante el crecimiento la mayor proporción de calcio de la dieta es utilizado para la formación y desarrollo óseo, otras funciones del calcio en el organismo animal incluyen la regulación de la contracción muscular, la trasmisión nerviosa, la coagulación sanguínea y la activación de sistemas enzimáticos (Vieira et al., 2009), en las aves adultas para la formación de la cáscara del huevo. Según Ahmed et al., (2013), la inclusión de diferentes fuentes de calcio incrementa la producción de huevos, el consumo de ración y mejora la conversión alimenticia.
En la avicultura, los requerimientos nutricionales de calcio considerados para la fabricación de los concentrados comerciales en Colombia se atienden según las recomendaciones del NRC (1994) de 2,5\%, valores que difieren de las recomendaciones de Rostagno et al., (2011) de 3,099\%, lo cual genera contraste en la formulación de la ración. Con base en lo expuesto anteriormente, el objetivo de este trabajo fue evaluar el efecto de los niveles de calcio en la dieta sobre el rendimiento zootécnico de codornices en postura.

\section{Materiales y métodos}

En la Unidad de Codornices de la Universidad de los Llanos, Villavicencio, Colombia, ubicada a 400 de altitud, $27^{\circ} \mathrm{C}$ de temperatura y $82 \%$ de humedad relativa, fue realizado un experimento dosis-respuesta, durante 26 semanas, en un diseño completamente al azar con cuatro tratamientos seis repeticiones y 10 aves por unidad experimental para un total de 240 aves de 75 días de edad y $151,9 \pm 8,5 \mathrm{~g}$ de peso.

Las aves estaban alojadas en un galpón cerrado con techo de eternit, ubicadas en baterías de jaulas, elaboradas con alambre galvanizado, de cinco pisos con tres divisiones y capacidad de 10 aves por división, dotados de comederos convencionales y bebederos automáticos.

Los tratamientos fueron constituidos por raciones isoprotéicas, isoenergéticas c ingredientes convencionales (tabla 1), con cuatro niveles de suplementación de carbonato de calcio $(4,9 ; 5,28 ; 5,67$ y $6,06 \%)$ resultando en dietas con concentración de 2,30; 2,45; 2,60 y $2,75 \%$ de calcio para codornices japonesas en fase de postura, siendo las raciones de presentación en forma de harina y formuladas para atender los requerimientos de lisina y metionina digestibles recomendados por Rostagno et al., (2011) y de proteína bruta determinados por Hurtado et al., (2013). 
El manejo diario de los animales consistió en el suministro de ración dos veces al día a las 07:00 y 16:00 horas para garantizar consumo de ración y de agua a libertad, la recolección y pesaje de huevos se realizó en horas de la mañana, lo mismo que la limpieza de instalaciones y del equipo, monitoreo de temperatura, humedad relativa, de estado sanitario de los animales y diligenciamiento de registros de producción, las heces fueron sometidas a compostaje.

Los parámetros zootécnicos evaluados fueron producción de huevos (ave/día), peso del huevo (g), consumo diario de ración $(\mathrm{g})$, conversión alimenticia (kg de ración/docenas de huevos y masa de huevo).

Los ingredientes para las dietas fueron analizados en el Laboratorio de Nutrición Animal de la Universidad de los Llanos.

\section{Análisis estadístico}

Los datos fueron recopilados en registros manuales diseñados para la recolección de información diaria, luego agrupados en planillas Excel y posteriormente sometidos análisis de varianza y de regresión polinomial en el programa SAEG (2007).

Las variables producción de huevos, consumo de ración, peso del huevo, conversión alimenticia y de calidad del huevo serán ajustadas por el modelo siguiente:

$Y=\beta_{0}+\beta_{1} \mathrm{Ca}+\beta_{2} \mathrm{Ca}^{2}+\varepsilon$ donde:

$Y=$ Valor de la variable dependiente (Producción de hue vos, consumo de ración, peso del huevo y conversión alimenticia)

$\beta_{0}=$ Constante de regresión (intercepto de la recta con el eje $Y)$

$\beta_{1}=$ Coeficiente de regresión del componente linear

$\beta_{2}=$ Coeficiente de regresión del componente cuadrático.

Min $=$ Niveles de calcio en la dieta

$\varepsilon=$ Error o desvío asociado a la distancia entre el valor observado $Y$ y el valor estimado $\hat{Y}$ de la ecuación ajustada.

Tabla 1. Composición centesimal de la dieta

\begin{tabular}{|l|c|c|c|c|}
\hline & \multicolumn{4}{|c|}{ Niveles de calcio, $\%$} \\
\hline & $\mathbf{2 , 3 0}$ & $\mathbf{2 , 4 5}$ & $\mathbf{2 , 6 0}$ & $\mathbf{2 , 7 5}$ \\
\hline Maíz & 56,772 & 56,772 & 56,772 & 56,772 \\
\hline Torta de soya & 31,98 & 31,98 & 31,98 & 31,98 \\
\hline Fosfato Bicalcico & 1,34 & 1,34 & 1,34 & 1,34 \\
\hline Carbonato de calcio & 4,90 & 5,28 & 5,67 & 6,06 \\
\hline Aceite vegetal & 1,61 & 1,61 & 1,61 & 1,61 \\
\hline Suplemento de vitaminas ${ }^{1}$ & 0,5 & 0,5 & 0,5 & 0,5 \\
\hline Sal & 0,45 & 0,45 & 0,45 & 0,45 \\
\hline L-lisina ${ }^{2}$ & 0,05 & 0,05 & 0,05 & 0,05 \\
\hline DL Metionina ${ }^{3}$ & 0,17 & 0,17 & 0,17 & 0,17 \\
\hline Material inerte & 2,228 & 2,024 & 1,644 & 1,264 \\
\hline Composición Estimada & & & & \multicolumn{2}{c|}{19,16} \\
\hline Proteína Bruta, \% & 19,16 & 19,16 & 19,16 & 2800 \\
\hline EM, kcalEM/kg & 2857 & 2800 & 2800 & 2,75 \\
\hline Calcio & 2,30 & 2,45 & 2,60 & 0,35 \\
\hline Fósforo disponible & 0,35 & 0,35 & 0,35 & 1,0 \\
\hline Lisina digestible & 1,0 & 1,0 & 1,0 & 0.45 \\
\hline Metionina digestible & 0.45 & 0.45 & 0.45 & \\
\hline
\end{tabular}

1 Composición del producto por kg: Vit A, 40.000 .000 UI; vit D3, 8.000.000 UI; vit K3, 10g; vit E 40g; vit B1, 4g; vit B12, 60mg; ácido fólico, 1,2g; ácido pantoténico; $32 \mathrm{~g}$; niacina $100 \mathrm{~g}$; excipiente CS 1,000g

2 L-lisina $78,5 \%$

3 DL-metionina $99 \%$ 


\section{Resultados y discusión}

Los resultados del efecto de las niveles de calcio sobre los parámetros productivos de codornices en postura se presentan en la tabla 2.

La producción de huevos se incrementó de forma cuadrática $(P=0,03)$ con dietas hasta con $2,45 \%$ de calcio y el consumo de ración se incrementó $(\mathrm{P}=0,04)$ con los niveles 2,45 y $2,75 \%$ de calcio. Sobre la conversión alimenticia de kg de ración/docenas de huevos, el peso del huevo y la conversión de $\mathrm{kg}$ de ración $/ \mathrm{kg}$ de huevo no hubo efecto $(P>0,05)$ de los niveles de calcio.

Al desarrollar la ecuación de regresión se estima en $2,48 \%$ el nivel de calcio para codornices japonesas en fase de postura, asumiendo un consumo de ración diario promedio de $24,5 \mathrm{~g}$, corresponde a consumo de $607 \mathrm{mg} /$ día/ave de calcio, estimativa que difiere de lo recomendado por Talamas (2012) y Vieira et al., (2012) de $2,80 \%$ y $647 \mathrm{mg} ; 2,0 \%$ y $555,4 \mathrm{mg}$ de calcio por ave día respectivamente.

El valor de calcio estimado es próximo al recomendado por Amoah et al., (2012) de 3,0 de calcio y postura entre $84-88,04 \%$, al de NRC (1994) de 2,5\% de calcio en la dieta. Los datos de este trabajo difieren de los Rostagno (2011) de 3,01\% y de los obtenidos por Costa et al (2010) de 3,5\% para codornices en el tercio final de postura (45-57 semanas de edad), lo que corresponde a un consumo diario de calcio de $899 \mathrm{mg}$.

Del mismo modo, Brandão et al., (2007) determinaron efecto cuadrático de los niveles de calcio sobre la producción de huevos, siendo 3,51\% de calcio el valor recomendado para optimizar los índices de desempeño en codornices de 46 a 130 días de edad. Los resultados obtenidos en este trabajo corroboran lo propuesto por Ahmed et al., (2013), que indican que el calcio en la dieta incrementa la producción de huevos. Valores relativamente altos son tolerados por la codornices en fase de postura, según Souza et al., (2016) niveles de calcio de hasta de 2,85 no influyen en los parámetros productivos, mejoran la calidad de la cáscara y mantienen la calidad interna del huevo.

El consumo diario de ración se incrementó de forma cuadrática $(\mathrm{P}=0,04)$ hasta el nivel de $2,75 \%$ de calcio, siendo el resultado inferior al constatado por Costa et al (2010) de 25,67 - 27,59 g/día y por Vieira et al (2012) de 28,06 g/ave/día. Los resultados de consumo superan los valores determinados por Amoah et al., (2012) de 21,46 - 23,47 g/día y el consumo encontrado por Talamas (2012) de 23,12 g/ave/día en promedio con niveles de 2,$8 ; 3 ; 3,2$ y 3,4\% de calcio en la dieta, las diferencias en los resultados citados se pueden explicar por la fase de postura del ave, dado que corresponden a condiciones experimentales con aves de diferentes edades. El mayor consumo de alimento coincide con el mejor resultado de producción de huevos lo que corrobora que aves regulan el consumo con la satisfacción de los requerimientos para máxima producción y que el calcio estimula el mayor desempeño de las aves, la regulación en el consumo de calcio coincide con la formación de la cáscara (Calderano, 2010).

La conversión alimenticia de docenas de huevo por kg de ración es próxima a la constatada por Talamás (2012) de 0,34, superando los datos reportados por Costa et al., (2010) de 2,77; 2,73; 2,63 y 2,65 para niveles de 2,0 2,5 3,0 3,5 respectivamente. Por otro lado, Amoah et al., (2010) y Brandão et al., (2007) obtuvieron mejor conversión alimenticia, que además, verificaron efecto cuadrático de los niveles de calcio para las variables masa de huevo y producción diaria de huevos.

Tabla 2. Rendimiento de codornices con diferentes niveles de calcio en la dieta.

\begin{tabular}{|c|c|c|c|c|c|c|}
\hline & \multicolumn{4}{|c|}{ Niveles de calcio } & \multirow[b]{2}{*}{$(\mathbf{P})$} & \multirow[b]{2}{*}{ CV } \\
\hline & 2,30 & 2,45 & 2,60 & 2,75 & & \\
\hline Producción de huevos, $\%{ }^{1}$ & 80,19 & 81,76 & 79,15 & 71,67 & 0,03 & 4,39 \\
\hline Consumo diario de ración, $\mathrm{g}^{2}$ & $24,57 \mathrm{~b}$ & $24,85 \mathrm{a}$ & $24,49 \mathrm{~b}$ & $24,84 \mathrm{a}$ & 0,04 & $\mathrm{O}, 63$ \\
\hline Peso del huevo, $\mathrm{g}^{3}$ & 11,02 & 10,38 & 10,33 & 10,29 & 0,44 & 3,56 \\
\hline Conversión Alimenticia, Docenas de huevos/kg de ración ${ }^{3}$ & 0,362 & 0,360 & 0,375 & 0,369 & 0,30 & 5,55 \\
\hline Conversión Alimenticia de kg de huevo/kg de ración ${ }^{3}$ & 2.77 & 2,89 & 3,02 & 2,98 & 0,30 & 5,53 \\
\hline
\end{tabular}

1 Efecto cuadrático, $Y=-0,3386 x^{2}+1,6817 x-1,2734, R^{2}=0,92$

2. $\mathrm{P}<0,05$

$3 P>0,05$ 
Los valores obtenidos de conversión alimenticia por masa de huevo difieren de los hallazgos de Costa et al., (2010), Vieira et al., (2012) y de Talamás (2012), que obtuvieron mejores resultados, aunque no constataron diferencias significativas de los niveles de calcio en la dieta sobre las variables en mención.

\section{Conclusiones}

El nivel estimado de calcio que mejora la producción de huevos de codornices recomendado es de 2,48\%.

\section{Bibliografía}

Ahmed NM, Abdel AKA, Elamin KM, Dafalla KY, Malik EE, Dousa BM. Effect of dietary calcium sources on laying hens performance and egg quality. J. Anim. Prod. Adv. 2013;3(7):226-231.

Amoah JK, Martin EA, Barroga AJ, Garillo EP, Domingo I. Calcium and phosphorus requirements of Japanese quail layers. Journal Applied. Biosciences 2012;54:3892-3900.

Brandão PA, Costa FGP, Silva JHV. Brandao JS, Nobre JGS. Goulart CC Exigência de cálcio para codornas japonesas (coturnix coturnix japonica) em postura. Acta Scientiarum. 2007;29(1):1721.

Calderano AA. Fracionamento de dietas com níveis diferenciados de cálcio e fósforo para aves de postura. Rev. Eletrônica Nutritime. 2010;7(5):1346-1352.

Costa CHR, Barreto SLT, Umigi RT, Lima HJA, Araújo MS, Medina P. Balanço de cálcio e fósforo e estudo dos níveis desses minerais em dietas para codornas japonesas (45 a 57 semanas de idade). Rev. Bras. Zootec. 2010;39(8):1748-1755.

Dudosola IO. Comparative evaluation of internal and external qualities of eggs from quail and guinea fowl. International Research Journal of Plant Science. 2010;1(5):112-115.
Fugikura WS. Situação e perspectivas da coturnicultura no Brasil. SIMPÓSIO INTERNACIONAL DE COTURNICULTURA, 1., 2002, Lavras. Anais... Lavras: Universidade Federal de Lavras, 2002. p.10.

Vieira DVG, Barreto SLT, Valeriano MH, Jesus LFD, Silva LFF, Mencalha R, Barbosa KS, Mendes RKV, Cassuce MR, Melo,TS. Requirements for calcium and phosphorus available for Japanese quail of the 26 to 38 weeks of age, Rev Bras Saúde Prod Anim. 2012;13(1):204-213.

Hurtado Nery VL, Torres NDM, Ocampo DA. Efecto de los niveles de proteína sobre el desempeño de codornices japonesas en fase de postura. Orinoquia. 2013;17(1):32-39.

National Research Council, NRC. (1994). Nutrient requirements of poultry.Washington, D.C. Pp.44-45.

Rostagno HS, Albino LFT, Donzele JL, Gomes PC, Oliveira RF, Lopes DC, Ferreira AS, Barreto SLT. (2011). Tabelas Brasileiras para aves e suínos. Composição de alimentos e exigências nutricionais. 2 ed. Viçosa:UFV, p. 186.

Sakomura NK, Rostagno HS. (2007). Métodos de pesquisa em nutrição de monogástricos. Jaboticabal: UNESP. p.283.

Silva RM, Furlan AC, Ton APS, Martins EN, Scherer C, Murakami AE. Exigências nutricionais de cálcio y fósforo de codornas de corte em crescimento. R Bras Zootec. 2009;38(8):1509-1517.

Souza DS, Calixto LFL, Lemos MJ, Silva Filho CA, Pinho TP, Machado CA, Melo IA, Togashi CK. Desempenho e qualidade de ovos de codornas em final de produção alimentadas com níveis crescentes de cálcio. Semina: Ciências Agrárias. 2016;37(4)sup 1:2395-2406. DOI: 10.5433/1679-0359.

Talamás SP. (2012). Níveis de cálcio para codornas japonesas em postura. Tese (Bacheralado em zootecnia), Universidade Federal de Roraima, 35p. 